\title{
Experimental Investigation of an Index-Mismatched Multiphase Flow Using Optical Techniques
}

\author{
H. Coronado Diaz and Ronald J. Hugo \\ Mechanical \& Manufacturing Engineering, University of Calgary, 2500 University Drive NW, Calgary, AB, Canada T2N 1N4 \\ Correspondence should be addressed to H. Coronado Diaz; hcoronado@gmail.com
}

Received 27 July 2012; Revised 18 December 2012; Accepted 1 January 2013

Academic Editor: Mina Hoorfar

Copyright (C) 2013 H. Coronado Diaz and R. J. Hugo. This is an open access article distributed under the Creative Commons Attribution License, which permits unrestricted use, distribution, and reproduction in any medium, provided the original work is properly cited.

\begin{abstract}
An experimental investigation of multiphase flow involving a liquid (water) and a gas (air) is performed. The results for three different scenarios are presented: fixed bubble, ascending bubble, and dispersed-bubble turbulent pipe flow. This study involves a comparison of statistical data collected using two sensing systems, a wavefront sensor and a high-speed video camera. A signal analysis technique based on signal attenuation is developed for data collected using the wavefront sensor. The three experiments performed provide experimental evidence that the Shack-Hartmann wavefront sensor, operating on signal attenuation, is a viable method for the study of multiphase bubble flows.
\end{abstract}

\section{Introduction}

Multiphase flow is defined as the simultaneous flow of several phases, with the simplest case being two-phase flow. The flow of two phases is found in many industrial processes including chemical and nuclear reactors, distillation towers, pipeline transport, injection of fluids for secondary recovery of oil and geothermal power plants.

One form of two-phase flow is gas-liquid dispersedbubble turbulent pipe flow. This flow is of significant importance in chemical and petroleum process industries where the interfacial area between phases needs to be large for improved process efficiency. Bubble size, velocity of bubbles, and their distribution along the pipe are all important parameters for optimizing the efficiency of processes that rely on dispersedbubble turbulent pipe flow.

Noninvasiveness is a desired attribute in any multiphase flow measurement technique given that the interference between the flow and the measurement device can affect the measured values. Many of the existing multiphase flow measurement techniques rely on the ability of the sensing apparatus to discriminate between certain physical properties that vary between phases, such as electromagnetic radiation attenuation or electrical impedance.
A large number of investigations reported over the last fifteen years describe the application of nonintrusive flow sensing techniques applied to the measurement and the study of multiphase fluid flows [1]. Nonintrusive measurement techniques typically provide either integral or line-integrated information, with the most common consisting of capacitance [2], conductance [3], ultrasound [4], and gammaradiation absorption [5] measurements. Both integral and line-integrated information collected from multiple sensors is often transformed into spatial information through the use of tomographic reconstruction algorithms.

The long-term goal of a larger research program is to apply optical tomography to the investigation of multiphase pipe flow. The multiple projection line-integrated data collected in the optical tomography system is obtained using Shack-Hartmann wavefront sensors (SH-WFS) [6]. The SHWFS is able to provide information about both strong indexof-refraction variations (gas-liquid or solid-liquid interfaces), as performed in the current investigation, as well as weaker liquid-liquid interfaces. An example of a flow with weaker liquid-liquid interfaces is a continuous-phase water-glycerine mixture with an immiscible dispersed-phase mineral oil. Through experimental design, the water-glycerine concentration can be altered so that the index mismatch between 
the water-glycerine mixture and the mineral oil is small. This results in a two-component system with weak index-ofrefraction variations. Consequently, the SH-WFS operates in a manner that is close to its original design [6].

A weak index-of-refraction system is not considered in the current investigation. What is performed here, rather, is an examination of the response of a single SH-WFS to a flow for which the SH-WFS has not been designed: a gas-liquid multiphase flow involving strong index-of-refraction variations ( $n \approx 1.33$ for water versus $n \approx 1.00$ for air). If the SHWFS can be demonstrated to provide meaningful quantitative data for a flow with strong index-of-refraction variation, then the use of SH-WFS sensors in optical tomography systems for the study of flows with both strong and weak indexof-refraction variations is supported. In order to investigate this further, it is first necessary to examine the performance of the SH-WFS when applied to a flow with strong indexof-refraction variation. In order to do this, three different but relatively well-understood gas-liquid multiphase bubble flows are considered.

This paper begins by describing the experimental apparatus and optical equipment used during this investigation. The response of the SH-WFS is investigated for the detection of both a single stationary air bubble and multiple ascending bubbles. This investigation is followed by a comparison of the performance of the SH-WFS to a high-speed video camera (HSVC) for detection of multiple ascending bubbles and for dispersed-bubble turbulent pipe flow. To conclude, the overall performance of the SH-WFS for application to indexmismatched multiphase flows is discussed.

\section{Experimental Apparatus}

Two different experimental systems are used during the investigation to examine the performance of the ShackHartmann wavefront sensor. In the first setup, a small vertical half-pipe section is used to examine the aberrating effects of both a single stationary bubble and of multiple ascending bubbles. The second system, a multiphase flow facility, is used to study the horizontal bubble flow under turbulent flow conditions. In both experimental systems, an acrylic optical contour is used to correct for the optical aberrations induced by the curvature of the water-filled pipe section [7].

2.1. Optical Diagnostics Equipment. The optical diagnostics used in the investigation consist of a Shack-Hartmann wavefront sensor (SH-WFS), and a high-speed video camera (HSVC). The HSVC provides the advantage of twodimensional images. Consequently, it also provides a good method by which the performance of the SH-WFS system can be examined when applied to a gas-liquid multiphase flow involving strong index-of-refraction variations.

2.1.1. Shack-Hartmann Wavefront Sensor (SH-WFS). The general arrangement of the SH-WFS with respect to both the half-pipe and the full-pipe is illustrated in Figure 1. A laser diode light source is expanded and collimated prior to transmission through the pipe section containing the aberrating multiphase flow. The emerging aberrated light transmits to the SH-WFS, which consists of a one-dimensional array of 64 cylindrical microlenses that focus the light onto a standard 2048 pixel line-scan camera (Dalsa Model CL-C4 2048 M). The analog readout of the line-scan camera is digitized using an Echotek VME-based A/D system with 8-bit resolution. Consequently, the readout data is quantified in terms of the A/D converter counts that range from 0 to 255 . Each microlens is $0.448 \mathrm{~mm}$ wide with a focal length of $37.45 \mathrm{~mm}$. The center of the focal spot generated by each microlens is quantified by examining the intensity profile of the 32 pixels that fill each subaperture (pixel size $=14 \mu \mathrm{m}$ ). The one-dimensional SH-WFS used in the investigation is capable of acquiring optical wavefronts at a temporal rate of 5,000 frames per second (fps).

Traditional Shack-Hartmann wavefront sensing applications involve the comparison of focal-spot centroidal locations produced by both a reference wavefront and an aberrated wavefront. The reference wavefront is often an unaberrated wavefront, produced by transmission through a medium without index-of-refraction variations. The change in the focal-spot centroidal location between the aberrated and unaberrated wavefronts is then computed for each subaperture, quantifying the distortion of the aberrated wavefront [8]. This method is based on the assumption that the focal-spot deflection in each subaperture will be less than one half of the number of pixels in a subaperture.

Given that the current investigation involves optical transmission through water continuous media $(n \approx 1.33)$ with dispersed air bubbles $(n \approx 1.00)$, strong index-ofrefraction variations are present resulting in large focalspot deflections. This paper demonstrates that the study of disperse bubble flow of water and air requires the use of an extinction-based approach rather than the standard method of SH-WFS processing.

2.1.2. High-Speed Video Camera (HSVC). The HSVC used during the experiments is a Photron Ultima 1024, capable of acquiring images at frame rates ranging from 60 to $16,000 \mathrm{fps}$. This camera is able to operate at a full $1024 \times 1024$ resolution at frame rates of up to $500 \mathrm{fps}$. The size of the pixel for this camera is $12 \mu \mathrm{m}$.

The HSVC enables images of the flow to be collected at high rates and then postprocessed for statistical quantities that can be compared to statistical data derived using the $\mathrm{SH}-$ WFS.

2.1.3. Optical Contour (OC). When using optical diagnostics to investigate pipe flow, special care needs to be taken to ensure that the strong optical aberrations induced by the fluid-filled pipe wall do not negatively impact the optical measurement being performed.

Prior to commencing this investigation, a custom-made corrective optic (referred to as an optical contour) was designed and manufactured using a computer numerically controlled (CNC) 5-axis machining station and used to correct for the aberrations induced by the water-filled acrylic pipe. The original design and manufacture of the optical 


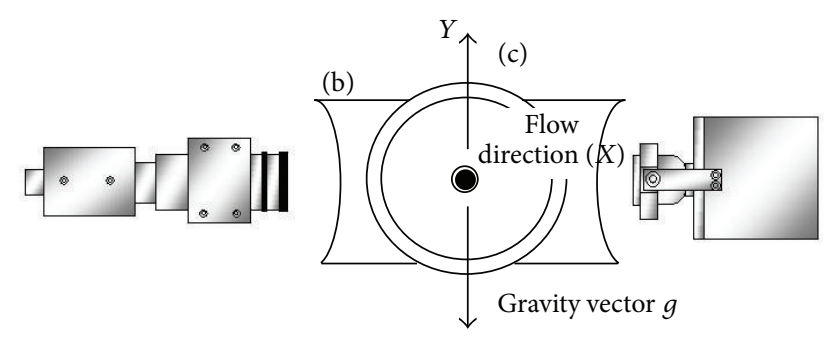

(a)

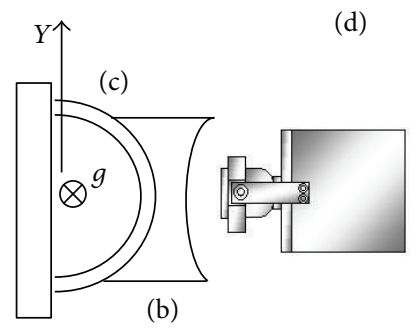

FIGURE 1: Schematic of the Shack-Hartmann wavefront sensor around the pipe and half-pipe: (a) laser-diode collimated light source, (b) optical contour, (c) pipe/half-pipe, and (d) 1D Shack Hartmann wavefront sensor.

contour is described in de Witt et al. [7]. The optical contour was designed to be clamped to the external pipe surface, making it relatively easy to attach it to different pipe locations. The optical contour was found to correct for the strong aberrations that would have otherwise been present while imaging through the water-filled pipe. The optical contour allows for approximately $80 \%$ of the central pipe to be investigated as shown in de Witt et al. [7].

The camera and the beam source are positioned perpendicular to the pipe axis as shown in Figure 1, with the spatial extent of flow investigated approximately $24.5 \mathrm{~mm}$ in the $y$-direction of the $57.15 \mathrm{~mm}$ internal diameter cast-acrylic pipe. As noted in Figure 1 (upper), two optical contours are required when applying the SH-WFS to the investigation of pipe flow. A replica of the CNC-manufactured optical contour described by de Witt et al. [7] is produced by first creating a mold of the CNC-manufactured optical contour and then pouring a time-hardening transparent urethane into the mold. The transparent urethane optical contour shows good optical characteristics when compared with the original acrylic lens. Moreover, it can be produced at a fraction of the cost and in less time than the CNC-manufactured optical contour.

2.2. Half-Pipe Apparatus. The half-pipe test section shown in Figure 1 (lower) enables the response of the optical diagnostic equipment to be examined while detecting air bubbles in water and in the absence of a net bulk velocity. The inner diameter of the acrylic half-pipe section is $57.15 \mathrm{~mm}$ with a wall thickness of $12.7 \mathrm{~mm}$. The half-pipe section is attached to an acrylic wall $11.4 \mathrm{~mm}$ in thickness. The optical contour is clamped to the outside of the acrylic half pipe, and given that one external surface was planar, the half-pipe apparatus requires only one optical contour.

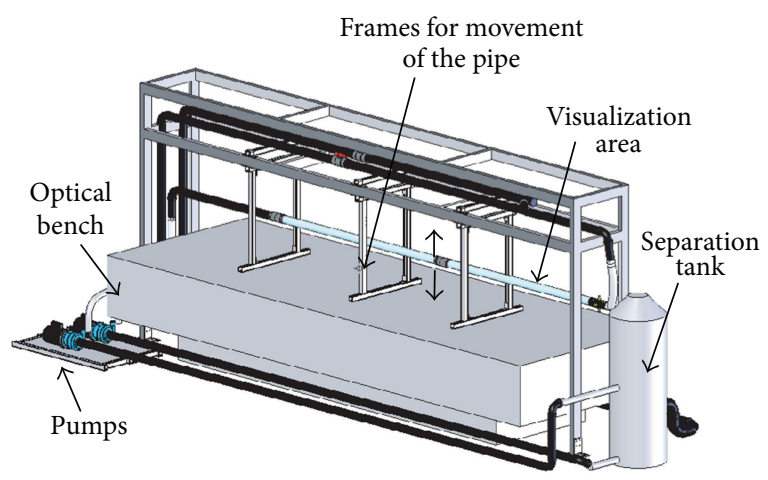

FIGURE 2: Description of the components of the flow facility.

Air bubbles are introduced into the half-pipe apparatus using two different methods. A single stationary bubble is introduced by injecting a known volume of air through a syringe into a small-diameter hose. A small-diameter hose is placed in the water-filled acrylic half pipe in a manner that ensures that the end of the hose is aligned with the sensing area of the SH-WFS.

For the generation of multiple ascending bubbles, a piece of porous material (sand packing of $2.54 \mathrm{~cm}$ length and $1.27 \mathrm{~cm}$ diameter) is connected to a regenerative blower (EG\&G Rotron Blower, with a maximum flow of $0.008684 \mathrm{~m}^{3} / \mathrm{s}$ and a maximum pressure of $61.0 \mathrm{~cm}$ of water) and placed at the bottom of the half pipe. The air supply hose connecting the blower to the sand packing has an internal diameter of $0.5 \mathrm{~mm}$ and is secured internally to the half pipe so as not to interfere with the measurement area $(2.54 \mathrm{~cm}$ wide) within the pipe.

2.3. Optically Active Multiphase Flow Facility. The multiphase flow facility was designed and built specifically to study dispersed multiphase flow using optical methods. The facility can operate using air, water, and oil; however, only air and water are considered in the current investigation.

A schematic of the multiphase flow facility is shown in Figure 2 with water and air as the operating fluids. The cast acrylic pipe section shown in Figure 2 runs the length of a $4.572 \mathrm{~m}$ by $1.829 \mathrm{~m}$ optical bench, enabling a wide variety of optical sensing methods to be applied to the study of multiphase flow. The facility can be broken down into five main sections as follows.

(1) Visualization Section. A $3.05 \mathrm{~m}$ long section of $5.72 \mathrm{~cm}$ inner diameter cast acrylic pipe with corrective optical contour forms the main diagnostic section.

(2) Pumping System. A centrifugal pump capable of delivering $7.25 \mathrm{~L} / \mathrm{s}$ at open flow is used to circulate the liquid phase. The air is supplied by a $3.7 \mathrm{~kW} \mathrm{2-}$ stage air compressor with $300 \mathrm{~L}$ vertical tank capable of delivering $8.5 \mathrm{~L} / \mathrm{s}$ at $689 \mathrm{kPa}$. The flow from the compressor is measured using a gas turbine flowmeter capable of sensing $1.9-28.3 \mathrm{~L} / \mathrm{s}$. 
(3) Piping. ABS black plastic pipe with $50.8 \mathrm{~mm}$ inner diameter is used for the fluid stream. Flow metering is performed on two straight section runs at the top of the flow facility. Flow rate for both water and oil is measured using a Panametrics UFP-1000 portable ultrasonic liquid flowmeter.

(4) Separation and Storage. A $196 \mathrm{~L}$ vertical cylindrical tank with a diameter of $45.72 \mathrm{~cm}$ is designed for the separation of water and air.

(5) Air Injection. A pipe of $38.1 \mathrm{~mm}$ diameter is connected to a $25 \mathrm{~mm}$ compressed air supply, and air injection is performed through a series of four $12.7 \mathrm{~mm}$ holes, each separated by $90^{\circ}$ around the perimeter of the $5.72 \mathrm{~cm}$ pipe.

\section{Shack-Hartmann Wavefront Sensor}

The performance of the SH-WFS is first examined by conducting experiments with a single bubble and with multiple ascending bubbles in the water-filled half-pipe.

3.1. Single Bubble Experiment. A single stationary bubble is introduced into the half-pipe apparatus by injecting a known volume of air (average volume $=0.0344 \pm 0.0035 \mathrm{~cm}^{3}$ ) through a syringe into a small-diameter hose. A smalldiameter hose is placed into the water-filled acrylic half-pipe so that the end of the hose is aligned with the sensing area of the SH-WFS.

The left part of Figure 3 shows a schematic of the incoming wavefront passing through a bubble where light encountering the bubble is shown to be scattered away from the detector. Results from a ray-tracing model (not shown) independently confirm this assumption. The right part of the same figure shows the corresponding intensity profile collected by the SH-WFS for a subset of the 2048 pixel array. Each $0.448 \mathrm{~mm}$ wide lens functions to focus the light into a relatively well-defined and quantifiable focal spot. Overlaid on the intensity profile is the reference measurement for the case of no bubble. By comparing the reference measurement to the single bubble measurement, it becomes apparent that the air bubble serves to scatter light away from the detectors, as depicted in the schematic to the left in the figure. The main consequence of this result is that it was not possible to apply the standard Shack-Hartmann data processing techniques that were described earlier. Rather, an alternate method of evaluating the data from the SH-WFS is required.

Due to low-signal levels, an evaluation that computes intensity attenuation is performed. To compare the intensities, the attenuation for each lens is calculated as the summed absolute difference between the reference intensity profile and the intensity profile collected with the air bubble in place. The attenuation is then passed through a thresholding algorithm that only accepts attenuations larger than 50 counts. A threshold of 50 counts is determined by quantifying noise as the summed absolute difference for multiple reference intensity profiles.

This thresholding algorithm is found to reject variations caused by electronic noise and results in clearer plots. The maximum value of attenuation is well above the threshold level and is greater than 1000 counts. The resulting plot in Figure 4 shows the existence of an aberration in lenses 37 through 46 (pixels 1168 to 1456). Given the 32 pixels per lenslet and a pixel width of $14 \mu \mathrm{m}$, the radius of the single bubble aberration is quantified to be $0.4032 \mathrm{~cm}$. Knowing the average volume injected by the syringe and assuming that the bubble remains spherical, the average radius is computed to be $0.4038 \pm 0.0136 \mathrm{~cm}$ or a difference of $0.15 \%$.

In order to ascertain the benefits of using the SH-WFS for bubble measurements, Figure 5 shows the intensity profile obtained for a single stationary bubble while using a standard line-scan camera without lenslet array. Once again, data from a reference measurement collected for the case of no bubble is overlaid. Although an attenuation region caused by the bubble is evident, it is more difficult to visually perceive this region than for the case with the lenslet array presented in Figure 3. The pixel-by-pixel absolute difference between the Single Bubble Measurement and the reference measurement is calculated, thresholded, and plotted as a contour plot in Figure 6. Although a region of strong attenuation is noted from pixel 814 to pixel 1096, artifacts of the differencing and thresholding routine appear at pixels that are not aligned with the bubble, as noted specifically near pixels 725 and 1400 .

The bubble diameter can be calculated from Figure 6 as the length from pixel 814 to pixel 1096, resulting in a diameter of $0.3948 \mathrm{~cm}$. When compared with the $0.4038 \pm 0.0136 \mathrm{~cm}$ average bubble diameter, an error of $2.23 \%$ can be calculated. This error is slightly larger than that obtained while using the lenslet array. The main difference between the two results is attributed to the increased difficulty in the thresholding routine for the case without lenslet array.

A more severe threshold is applied to the case without lenslet array in order to filter the data. The contour plot of the differences between intensity profiles (Figure 6) results in increased noise near the edges of the bubble. The diameter of the single bubble is estimated with increased error from Figure 6 due to the inconsistency in light attenuation along the pixel array. In comparison, the results for the experiments with the lenslet array (cf. Figure 4) lead to a more uniform region for the single bubble experiment.

3.2. Multiple Ascending Bubbles Experiment. Data for multiple ascending bubbles in the half-pipe apparatus is collected at a line rate of $2.5 \mathrm{kHz}$, yielding 256 lines of data with 0.0004 seconds between samples. When multiple bubbles are studied, it is noted that clustering between two or more bubbles may be observed leading to an uncertainty in the response of the SH-WFS.

For the case of multiple ascending bubbles, the subaperture spot centroidal location does not shift significantly when compared to the unaberrated reference signal. Furthermore, the signal for multiple bubbles is not fully attenuated as it is for a single bubble. It is suspected that scattered light from one bubble may be detected by other subapertures not directly aligned with that bubble. Even though the amount of light extinction is less than that for the case of a single bubble, 

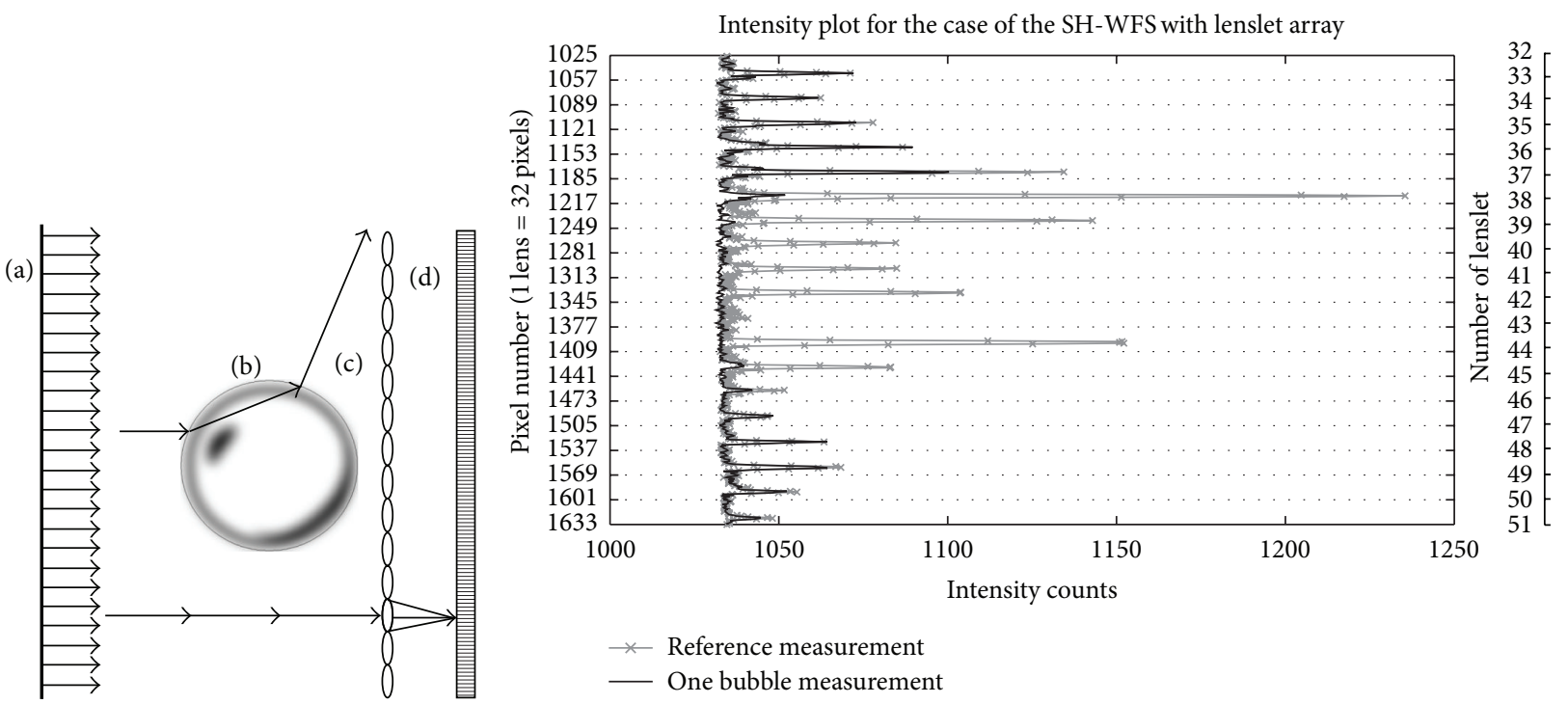

FIGURE 3: Scheme of the wavefront passing through the bubble in the half-pipe and plot with the intensity response from the wavefront sensor, (a) incoming wavefront, (b) single bubble, (c) lenslet array, and (d) pixel array.

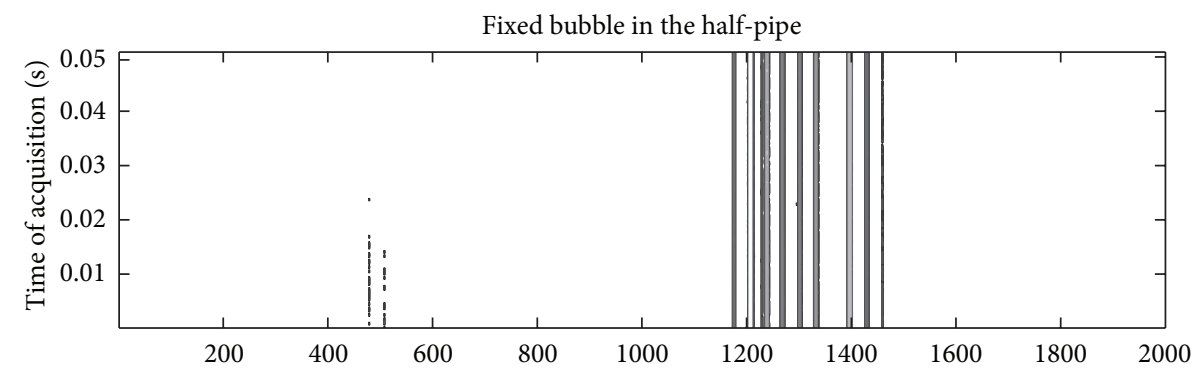

(a)

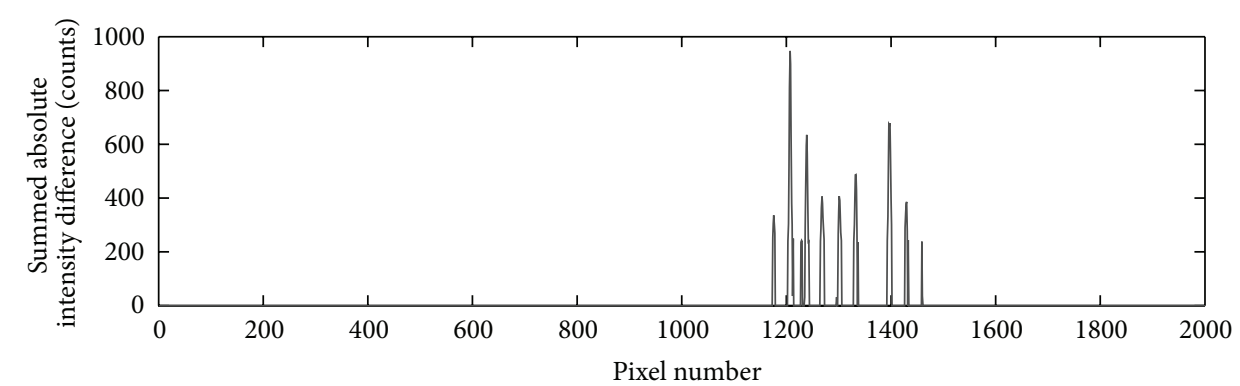

(b)

FIGURE 4: Contour of intensity difference with time for one bubble (a); profile of intensity difference at one time sample (b).

the extinction is still more quantifiable than a shift in spot centroidal location. Consequently, the same signal analysis routine as performed for the single bubble experiment is used, with results shown in Figure 7.

When the lenslet array is removed from the SH-WFS, the data collected is more difficult to interpret. Figure 8 shows an example of the datasets obtained for ascending bubbles without the lenslet array. It becomes evident that the lenslet array improves the ability to detect bubbles, especially while analyzing bubbles in motion. The behavior of the SH-WFS is examined in further detail in the next section when the SWWFS is compared to a HSVC.

\section{Shack-Hartmann Wavefront Sensor versus High-Speed Camera}

Two main experiments are performed involving the application of both the SH-WFS and the HSVC to the study of air bubbles in water. The first experiment involves ascending 

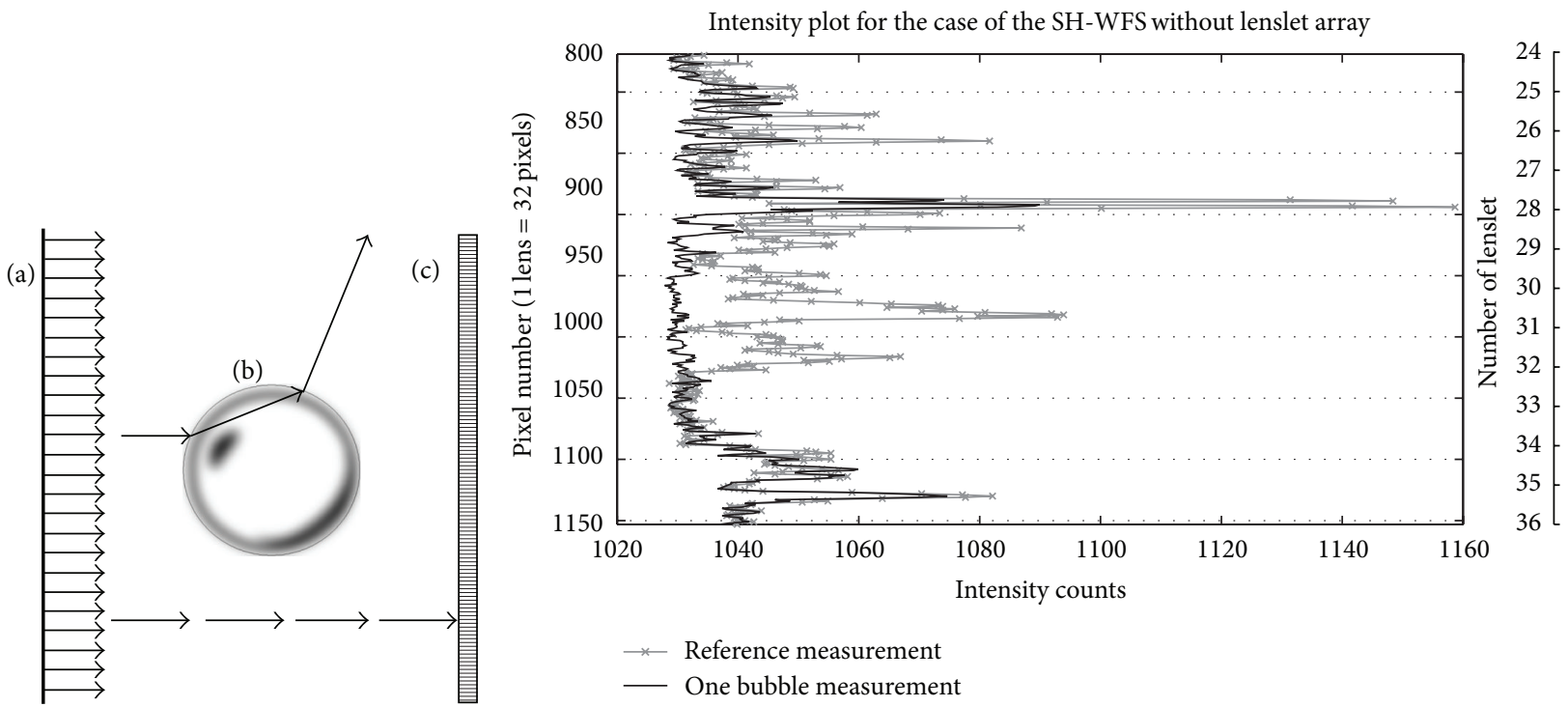

Figure 5: Schematic of the wavefront passing through the bubble in the half pipe and plot with the intensity response from the wavefront sensor, (a) incoming wavefront (b) single bubble (c) pixel array.

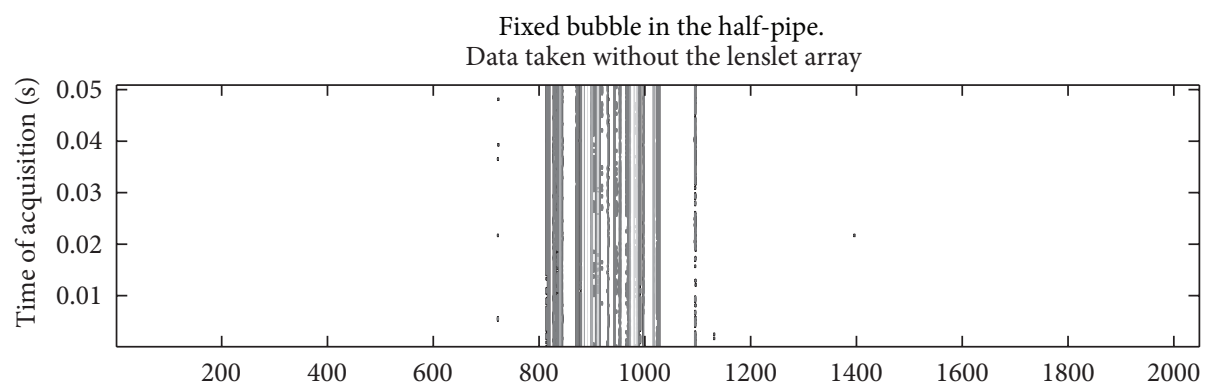

(a)

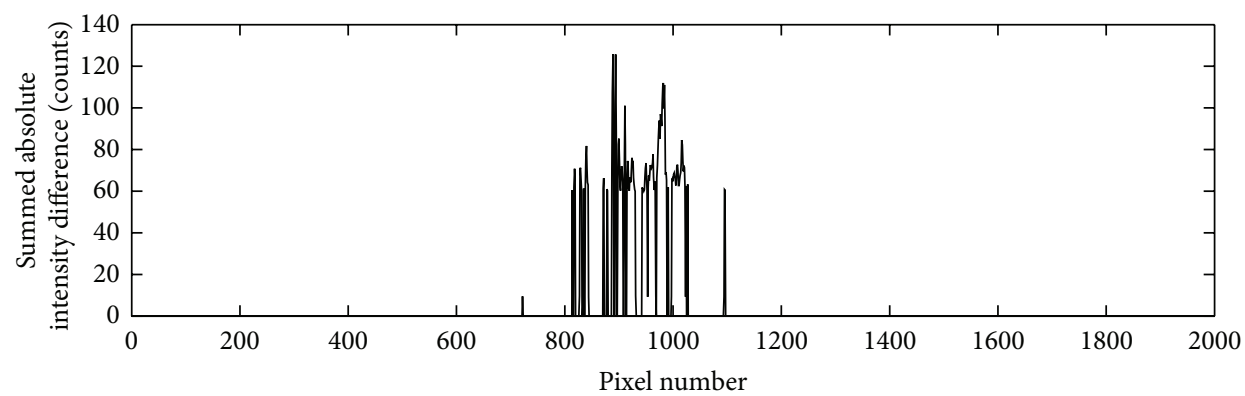

(b)

FIGURE 6: Contours of intensity difference with time for one bubble without lenslet array (a) and profile of intensity difference at one time sample without lenslet array (b).

bubbles in the water-filled half-pipe, and the second involves dispersed-bubble turbulent pipe flow.

4.1. Half-Pipe Experiment: Multiple Ascending Bubbles. The flow is analyzed using both the SH-WFS and the HSVC. The high-speed video data is collected at a frame rate of $2 \mathrm{kHz}$ with a resolution of 256 by 512 pixels. Figure 9 shows an example of the data taken with the HSVC for the ascending bubbles experiment. Each dataset collected using the HSVC consists of approximately 70 to 120 bubbles ascending through the viewing area, from which it is possible to extract statistical information for comparison with the SH-WFS. Bubble diameter is extracted from HSVC frames displaying spherical bubbles, while velocity is extracted for bubbles moving vertically upward without collision. By using the HSVC, the average diameter of the bubbles is estimated 


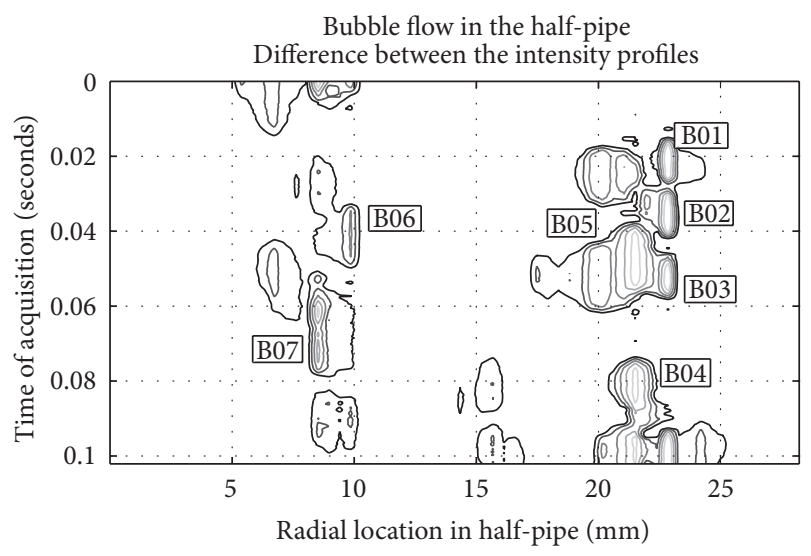

FIGURE 7: Variation in intensity measured for the bubble flow in the half-pipe.

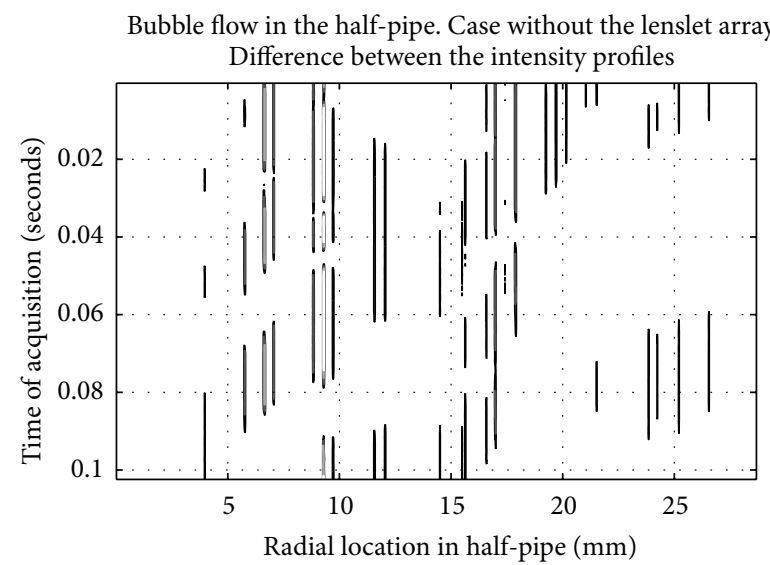

FIGURE 8: Variation in intensity measured for the bubble flow in the half-pipe without the lenslet array.

to be $2.33 \pm 0.5 \mathrm{~mm}$ and the average velocity of the bubbles is estimated to be $0.250 \mathrm{~m} / \mathrm{s}$.

Analysis of the SH-WFS data reveals the average diameter of the bubbles as $2.08 \pm 0.224 \mathrm{~mm}$ and the average velocity as $0.236 \pm 0.041 \mathrm{~m} / \mathrm{s}$. Consequently, both the average size and velocity collected using the SH-WFS falls within the range of values estimated using the HSVC. A more detailed comparison involving probability density functions for both sensors is shown in Figure 10 from which it is noted that similar distributions result for both sensing systems.

Processing data collected during the multiple ascending bubble experiment reveals the difficulties associated with either sensing system when multiple bubbles are present. For instance, in the case of results from the SH-WFS it is difficult to say what is happening when two contour peaks are very close together. This behavior may be attributed to two bubbles, with one behind the other, or to interactions between bubbles, such as coalescence. Data collected using the HSVC presents similar challenges, as denoted by the circles in Figure 9. Multiple bubbles challenge automatic bubble detection routines, forcing one to manually inspect images.

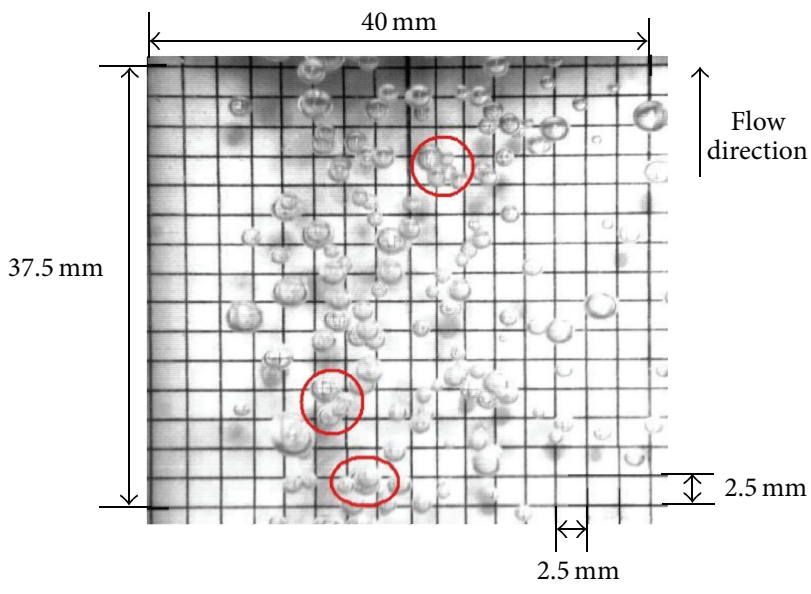

FIGURE 9: Data taken with the HSVC for the case of ascending bubbles in the half-pipe. The circles show clusters of bubbles observed.

4.2. Optically Active Multiphase Flow Facility. As in the case for the water-filled half-pipe, the bubble flow investigated using the multiphase flow facility is performed by collecting data using both the HSVC and the SH-WFS. The multiphase flow facility enables the horizontal turbulent pipe flow to be investigated. This type of flow behaves in a fundamentally different manner from the previously discussed ascending bubble flow. Due to the presence of strong shear forces, bubbles in the horizontal dispersed-bubble turbulent pipe flow are more likely to be ellipsoid in shape rather than spherical. Also, the flow of bubbles is affected by the velocity field of the continuous phase (carrying fluid), with bubbles moving slower along the walls and faster towards the centerline of the pipe. While comparing the two measurement methods, an analysis of bubble size, velocity, and position within the pipe is performed.

The water velocities that resulted in dispersed-bubble flow using the multiphase flow facility ranged from $1.31 \mathrm{~m} / \mathrm{s}$ to $1.82 \mathrm{~m} / \mathrm{s}$. Seven separate water velocities are investigated. Figure 11 shows an example of the bubble distributions in the pipe for each investigated velocity using the HSVC. A total of 1.024 seconds of data is collected with the HSVC for each water velocity at a frame rate of 1000 frames per second (1024 frames). Data collected using the HSVC is taken across the entire pipe diameter.

It is not possible to quantify the amount of air injected during these tests due to the air flow rates being below the sensing range of the gas turbine flow meter. The flow rate of injected air is, however, assumed to be constant for all water velocities investigated given that a fixed flow restriction is imposed to the air inlet hose for all cases.

Figure 12 shows data collected using the SH-WFS for each water velocity. A total of 15 SH-WFS data sets $(0.1024$ seconds each) are collected for a total of 1.54 seconds of data at each flow velocity. 


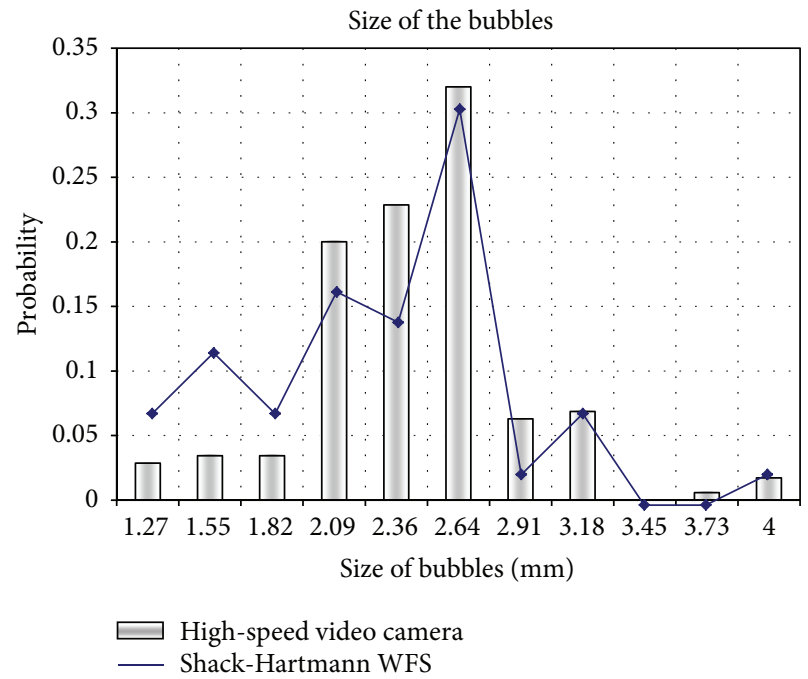

(a)

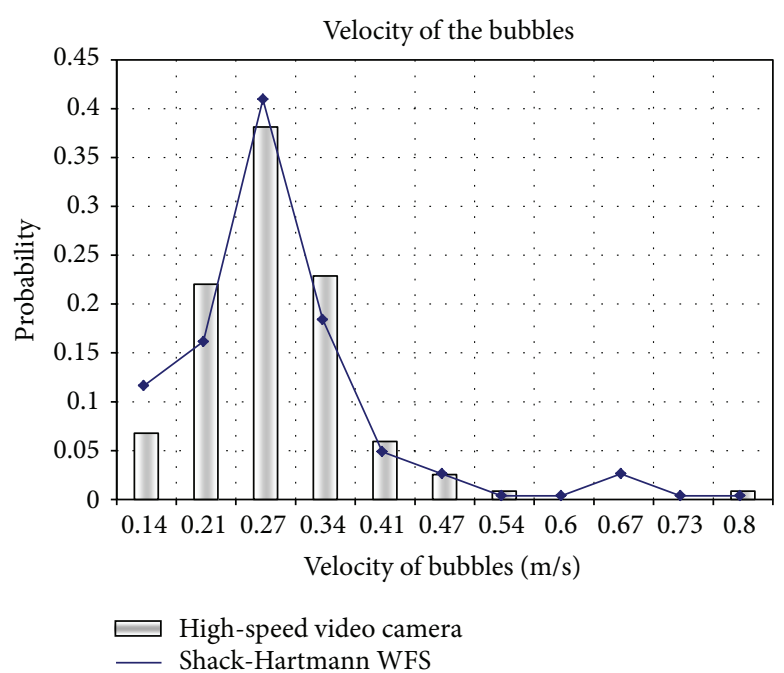

(b)

FIGURE 10: Statistical analysis for the diameter of spherical particles in the flow and velocity of the ascending bubbles.
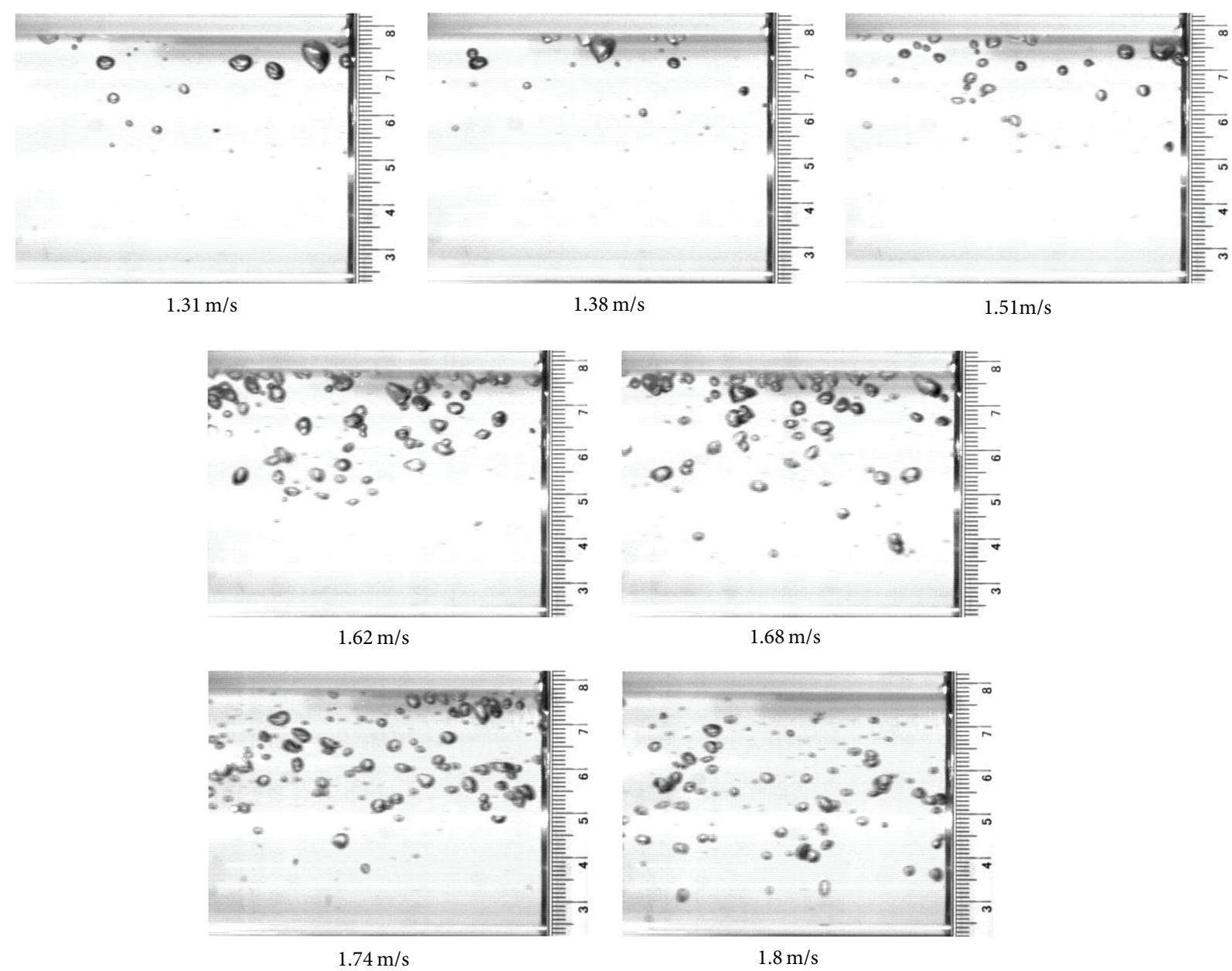

$1.74 \mathrm{~m} / \mathrm{s}$

FIGURE 11: Typical results from the HSVC for each water velocity sample. 
The SH-WFS is only able to detect across $24.5 \mathrm{~mm}$ of the $57.15 \mathrm{~mm}$ inner diameter cast acrylic pipe, and consequently it is necessary for the frame of reference of the SH-WFS detector to be aligned with the physical space of the pipe. This is performed by placing a dark object along the center of the pipe and by noting the subaperture (subaperture 18) where a loss of signal occurred. This subaperture is then referenced as the pipe centerline and labeled by a dark solid line in all plots. Even though the sensing length of the SHWFS is $28.67 \mathrm{~mm}$, only $24.5 \mathrm{~mm}$ of the sensing length results in meaningful intensity measurements. The optical contour causes a reduction of the collimated light at the entry of the pipe and magnification at the exit; this effect reduces the width of the collimated beam within the pipe by $5 \%$. For more details about the behavior of light through the optical contour, the interested reader is referred to de Witt et al. [7].

4.2.1. Data Processing. In order to compare HSVC data to SHWFS data, HSVC data is extracted only when a bubble crosses a reference line. Each bubble-crossing event yields data with regards to the following characteristics: the vertical position of the bubble in the pipe, the bubble size (both horizontal and vertical major axes), and the number of frames for the bubble to move through the reference line (bubble horizontal velocity). The use of a reference line to analyze data from HSVC enables a direct comparison to velocity data measured using SH-WFS.

Bubble size is measured with the help of a grid that is superimposed on each image. The grid that is used has a minimum size of $1 \mathrm{~mm}$ per cell. Approximately 350 bubblecrossing events are detected at the highest water velocities, while only 50 to 80 bubble-crossing events are detected at the lowest velocities.

Image analysis is not required for the SH-WFS data; however, as with the HSVC data, the SH-WFS data is also analyzed manually. The sizes and velocities are obtained from the summed absolute difference of intensity data. A similar grid to the one used with the HSVC is used to detect the size of the objects (bubbles) in the contour plots. The size of the bubbles is calculated by multiplying the size of the pixel in the sensor $(14 \mu \mathrm{m})$ by the maximum number of pixels in the contour plot. The time for a crossing event is calculated with the maximum length of the body in the contour plot (vertical axis). The convective velocities are computed by dividing each diameter by the corresponding time for a crossing. The position of each bubble inside the pipe is calculated by referencing the pipe centerline to the middle point of each bubble.

Given that the line-scan camera is a one-dimensional sensor, it is only possible to quantify bubble size in the crossflow direction. Consequently, the data collected with the SHWFS includes the vertical position of the bubble in the pipe, the size of the bubble (vertical axis dimension), and the number of frames (time) that it would take for each bubble to move through the sensing plane.

The image distortion effects due to the presence of the optical contour are also taken into account, and the data presented here is the corrected physical dimension. The magnification used for the optical contour is as reported in de Witt et al. [7].

4.2.2. Bubble Shape. Of the more than 1000 bubbles analyzed using the HSVC data, only $26 \%$ of the bubbles reveal spherical shape. The remaining bubbles are ellipsoidal in shape. An analysis of the shape of the bubbles is made using the data acquired with the HSVC and a ratio of bubble size in the flow direction $(X)$ to bubble size in the cross-flow direction $(Y)$ computed. A probability density function of bubble aspect ratio is shown in Figure 13. Three distributions are plotted, one with data for all water velocities and the other two with data from both the lowest and highest velocities. The bubbles become more ellipsoidal at the higher flow velocities, with the long axis oriented in the flow direction. The mean bubble size in the $X$ direction is $2.44 \mathrm{~mm}$ versus $2.14 \mathrm{~mm}$ in the $Y$ direction.

Investigations by Crowe et al. [9] and Hesketh et al. [10, 11] have reported a similar segregated distribution for bubble size in horizontal dispersed-bubble turbulent pipe flow.

This variation in bubble aspect ratio will affect velocity calculations made using the SH-WFS measurements given the assumption that bubbles are spherical. Bubbles are more likely to be elongated in the flow direction, resulting in a reduction in measured bubble velocity using the SH-WFS. The data presented in this paper is taken very close to the injection point which results in increased bubble shape variations. The assumption of sphericity has been made by other researchers investigating the horizontal bubble flow $[12,13]$ with the assumption most valid for fully developed flow with bubble diameter less than $2.0 \mathrm{~mm}$.

4.2.3. Bubble Size and Position. Figure 14 shows the data collected for the lowest and highest water velocities of bubble size versus pipe position using both the HSVC and the SHWFS. When comparing the behavior of bubble size versus pipe position at the lowest water velocities, the largest bubbles are observed towards the top of the pipe. As water velocity increases, the bubbles are seen to distribute more evenly across the entire pipe diameter.

A pattern is observed in the size information computed using the SH-WFS data where bubble size falls into discrete bands. These discrete bands result as the size calculation is performed based on the width of each lenslet, resulting in a resolution of $0.448 \mathrm{~mm}$ with an uncertainty of $\pm 0.224 \mathrm{~mm}$. When two bubbles are very close to each other or when the difference in intensity shows a non-regular shape towards the edge (due to noise from scattered light from another subaperture), a minimum size of half of a lenslet is assigned.

With the exception of the largest water velocity, the maximum bubble size for all data collected with the $\mathrm{SH}$ WFS is smaller than the maximum size calculated using the HSVC. The SH-WFS is also able to detect smaller bubbles than the HSVC. While small bubbles can be seen in the 

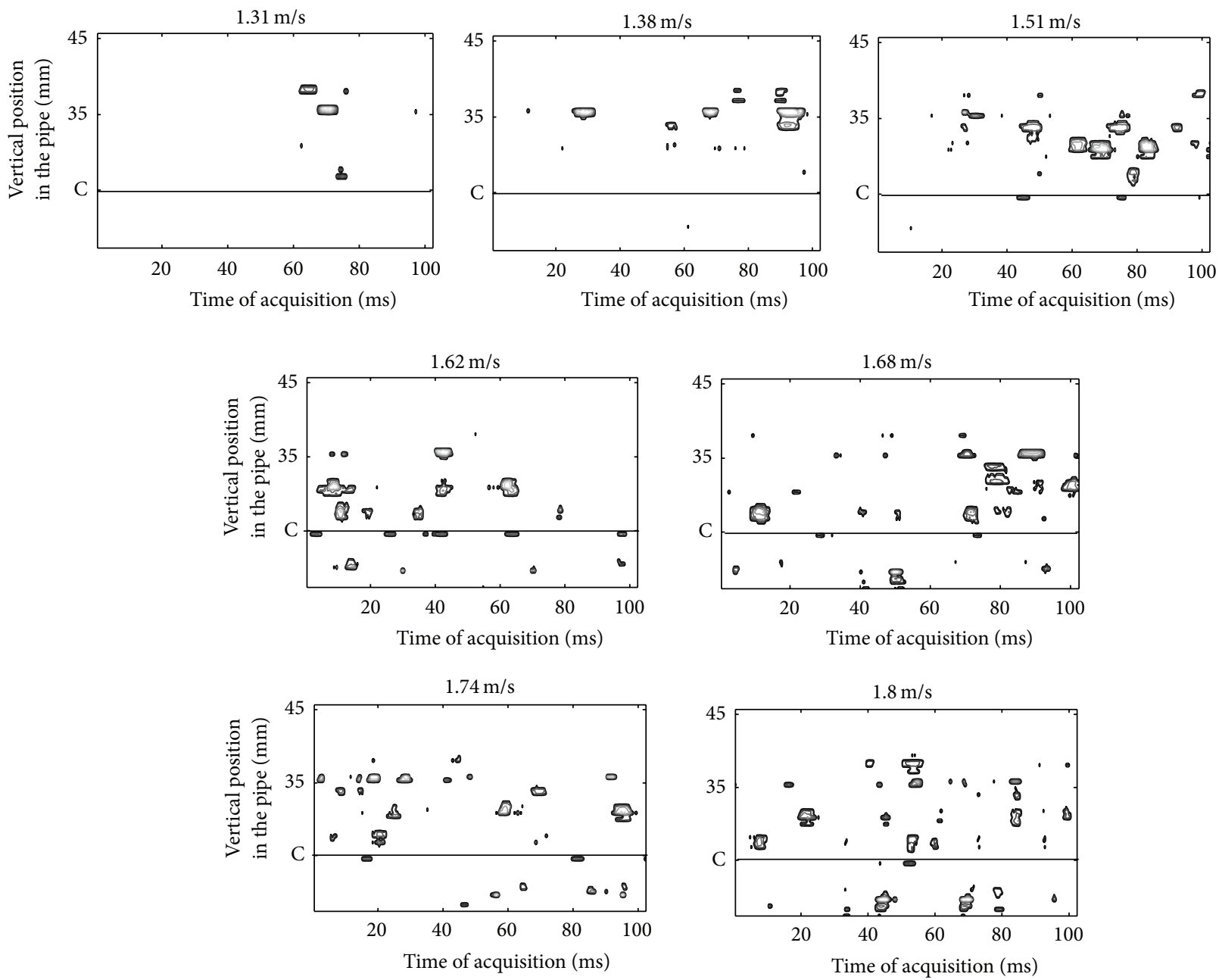

FIGURE 12: Typical results from the SH-WFS for each water velocity sample. Letter "C" in each plot represents the center of the pipe.

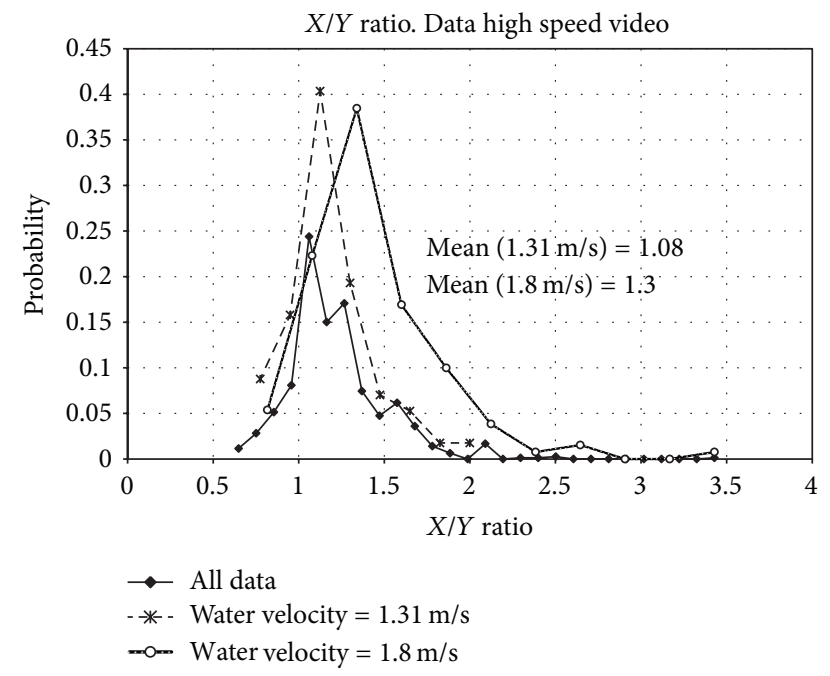

FIGURE 13: Analysis of the sphericity of bubbles with the high-speed video.

high-speed video, it is very difficult to obtain an accurate size calculation due to a limit in sensor resolution. The minimum size acquired for the SH-WFS was $0.224 \mathrm{~mm}$ while for the HSVC the minimum size is $0.54 \mathrm{~mm}$, more than double that of the SH-WFS. The cause for this difference is believed to be attributed to the depth of field of the HSVC. Bubbles outside the depth of field of the HSVC would be blurred, making it more difficult to resolve them. The SH-WFS, on the other hand, involves collimated laser light. Consequently, it does not have a depth of field but rather is able to detect bubbles independent of where they are located within the pipe. If the HSVC had a smaller field of view like the SH-WFS, the resolving capability of the HSVC would have improved. The number of pixels in the SH-WFS is also larger than that for the HSVC, which also results in the noted difference. Had a higher resolution HSVC been used, the results would have been more comparable.

4.2.4. Bubble Velocity and Position. A comparison of bubble velocity data with pipe position is shown in Figure 15. The distributions of velocities for both sensors show good agreement, with similar maximum velocities for both sensors. The analysis of longer data sets would help to reduce the scatter observed in the distribution for both size and velocity. 


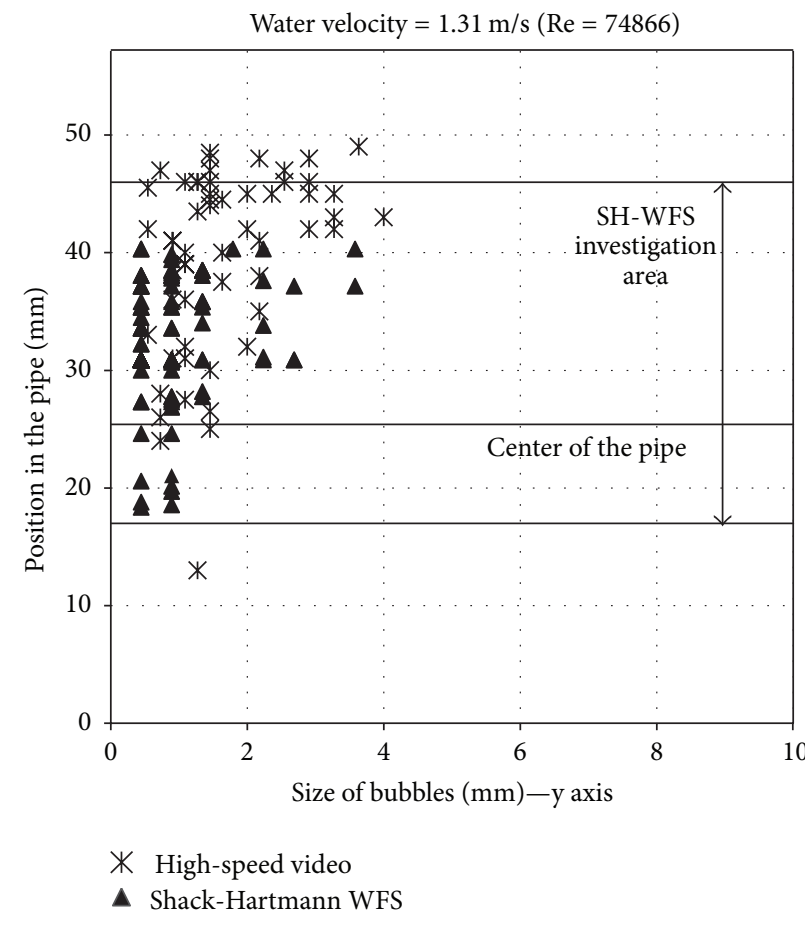

(a)

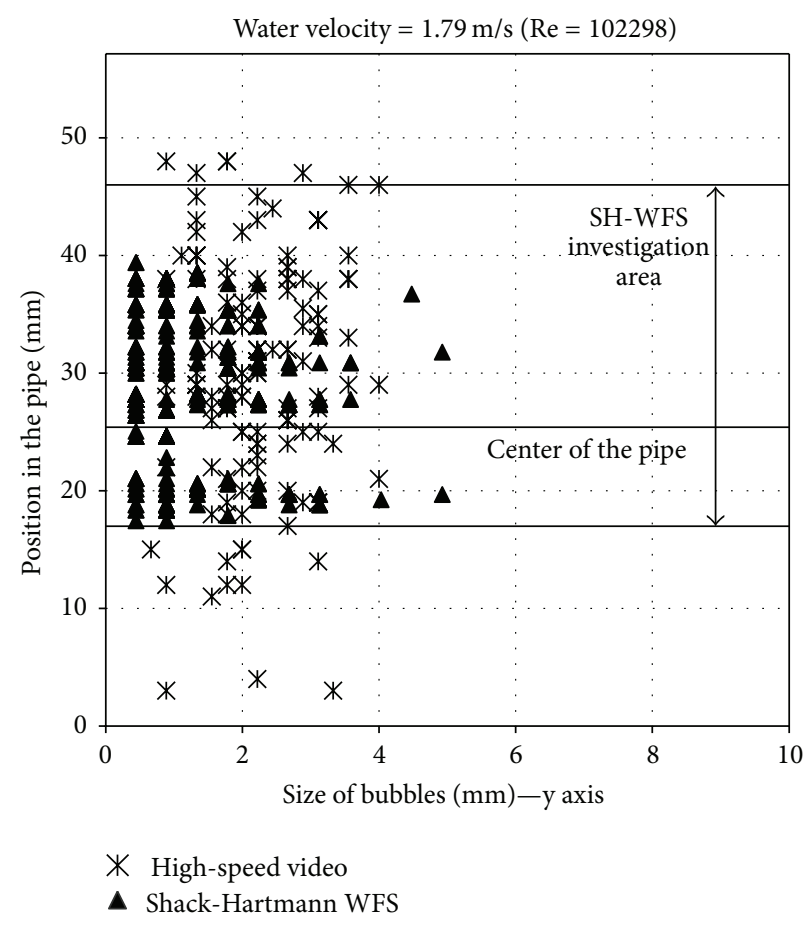

(b)

FIGURE 14: Comparison of position of bubbles in the pipe versus size of the bubbles.

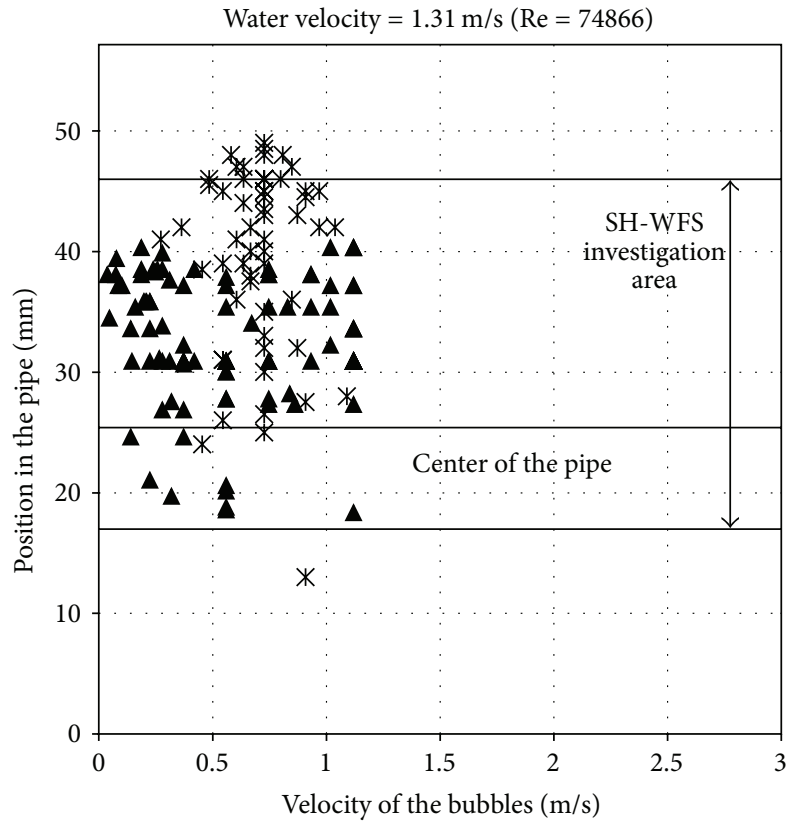

Ж High-speed video

A Shack-Hartmann WFS

(a)

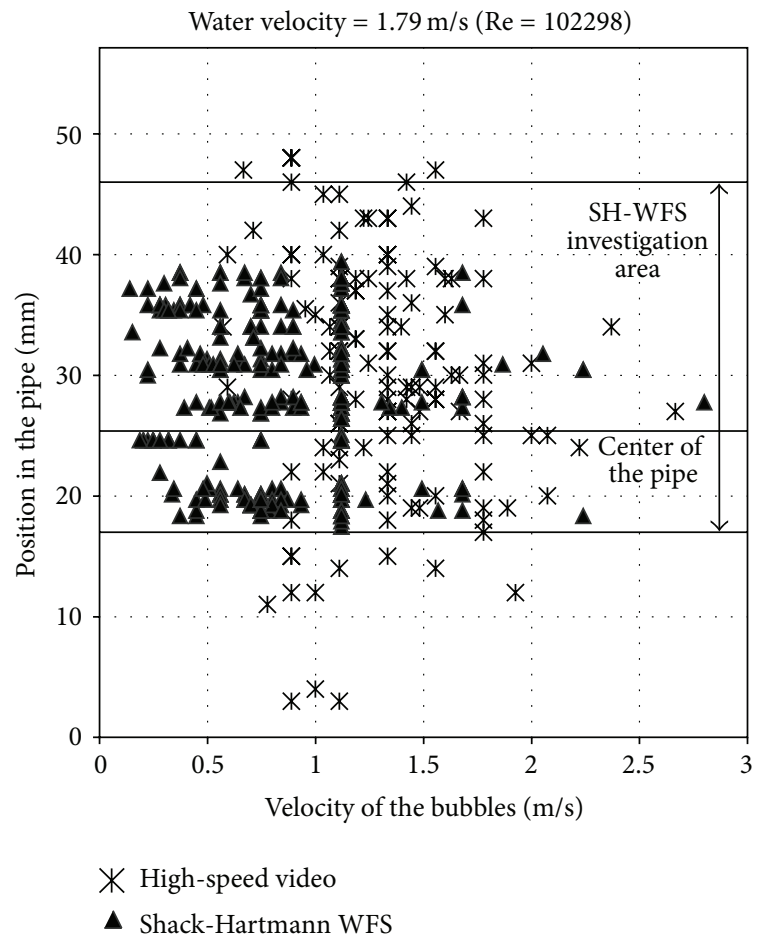

(b)

Figure 15: Comparison of position of the bubbles in the pipe versus velocity of the bubbles. 


\section{Conclusions}

This paper has investigated the performance of a SH-WFS for the analysis of dispersed-bubble turbulent flow. The presence of dispersed bubbles in the continuous water media is found to cause extinction of the collimated light source for the $\mathrm{SH}$ WFS.

A statistical comparison between the SH-WFS and HSVC reveals good agreement for two different experiments: ascending bubble flow and horizontal pipe flow. The depth of field of the HSVC makes it more difficult to detect small bubbles (less than $0.55 \mathrm{~mm}$ ) when compared to the SH-WFS, and consequently the SH-WFS has a better resolution than the HSVC for the optical configuration investigated. Had the HSVC had a smaller field of view or larger number of pixels, its resolution would have improved.

The three individual experiments that were performedsingle fixed bubble, multiple ascending bubbles, and horizontal dispersed-bubble turbulent pipe flow, all provide strong experimental evidence that the SH-WFS is a reliable method for the study of multiphase bubble flows. The SH-WFS has certain advantages/disadvantages when compared to other measurement systems.

In comparison to the HSVC, the SH-WFS has the advantage of being able to detect bubbles at any plane inside the pipe whereas bubble measurement using the HSVC was more dependent on the depth of field of the camera lens system. The bubble detection procedures for the SH-WFS are simpler than those for the HSVC, providing a computational advantage during postprocessing operations. Given that the SH-WFS is a one-dimensional sensor, it has the advantage of being able to store more frames and to collect them at higher frame rates. The one-dimensional nature of the SH-WFS does pose a disadvantage when compared to the HSVC, given that it is difficult to measure convection velocity without first having to make assumptions about bubble dimension in the flow direction (i.e., the spherical bubble assumption).

The SH-WFS has been shown to perform as an extinctionbased sensing technique when applied to a flow containing components with strong index-of-refraction variations, such as the liquid-gas flow investigated here. Additional sensing capability exists when the system is applied to flows with weak index-of-refraction variations, such as two immiscible fluids with minor index of refraction differences. This would enable the SH-WFS to detect both dispersed bubbles and oil droplets in a water-glycerin continuous flow, for instance. Exploration of this research topic has been left as a future investigation.

\section{Acknowledgments}

The authors would like to acknowledge the support of the Natural Sciences and Engineering Research Council of Canada (NSERC) through its Discovery Grant Program and to acknowledge the National Council of Science and Technology of Mexico (CONACyT) for its financial support.

\section{References}

[1] T. Dyakowski, "Process tomography applied to multi-phase flow measurement," Measurement Science and Technology, vol. 7, no. 3, pp. 343-353, 1996.

[2] S. M. Huang, A. B. Plaskowski, C. G. Xie, and M. S. Beck, "Tomographic imaging of two-component flow using capacitance sensors," Journal of Physics E, vol. 22, no. 3, pp. 173-177, 1989.

[3] F. J. Dickin, R. A. Williams, and M. S. Beck, "Determination of composition and motion of multicomponent mixtures in process vessels using electrical impedance tomographyI. Principles and process engineering applications," Chemical Engineering Science, vol. 48, no. 10, pp. 1883-1897, 1993.

[4] J. Wolf, "Investigation of bubbly flow by ultrasonic tomography," Particle and Particle Systems Characterization, vol. 5, no. 4, pp. 170-173, 1988.

[5] S. B. Kumar, D. Moslemian, and M. P. Duduković, "A $\gamma$-ray tomographic scanner for imaging voidage distribution in twophase flow systems," Flow Measurement and Instrumentation, vol. 6, no. 1, pp. 61-73, 1995.

[6] R. V. Shack and B. C. Platt, "Production and use of a lenticular Hartmann screen," Journal of the Optical Society of America, vol. 61, pp. 656-660, 1971.

[7] B. J. de Witt, H. Coronado-Diaz, and R. J. Hugo, "Optical contouring of an acrylic surface for non-intrusive diagnostics in pipe-flow investigations," Experiments in Fluids, vol. 45, no. 1, pp. 95-109, 2008.

[8] R. K. Tyson, Principles of Adaptive Optics, Academic Press, New York, NY, USA, 2nd edition, 1991.

[9] C. Crowe, M. Sommerfeld, and Y. Tsuji, Multiphase Flows with Droplets and Particles, CRC Press, New York, NY, USA, 1998.

[10] R. P. Hesketh, F. Russell, and A. W. Etchells, "Bubble size in horizontal pipelines," AIChE Journal, vol. 33, no. 4, pp. 663-667, 1987.

[11] R. P. Hesketh, A. W. Etchells, and T. W. F. Russell, "Experimental observations of bubble breakage in turbulent flow," Industrial and Engineering Chemistry Research, vol. 30, no. 5, pp. 835-841, 1991.

[12] P. Andreussi, A. Paglianti, and F. S. Silva, "Dispersed bubble flow in horizontal pipes," Chemical Engineering Science, vol. 54, no. 8, pp. 1101-1107, 1999.

[13] M. M. Razzaque, A. Afacan, S. Liu, K. Nandakumar, J. H. Masliyah, and R. S. Sanders, "Bubble size in coalescence dominant regime of turbulent air-water flow through horizontal pipes," International Journal of Multiphase Flow, vol. 29, no. 9, pp. 1451-1471, 2003. 

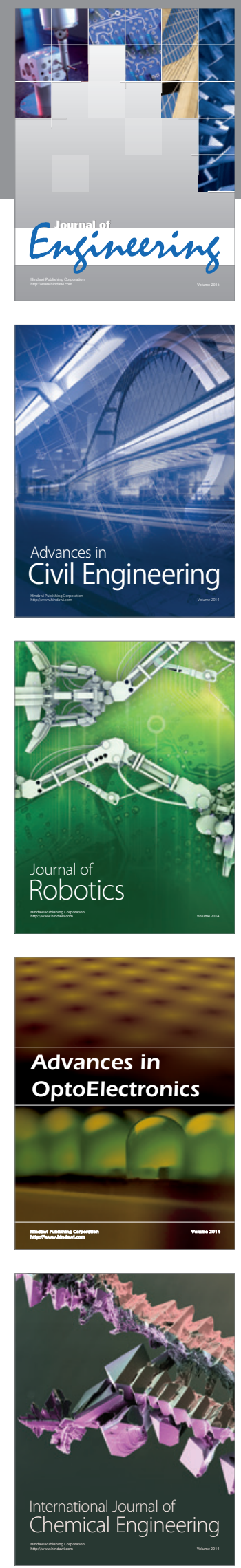

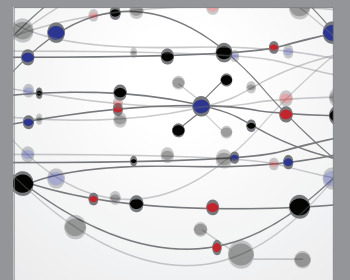

The Scientific World Journal
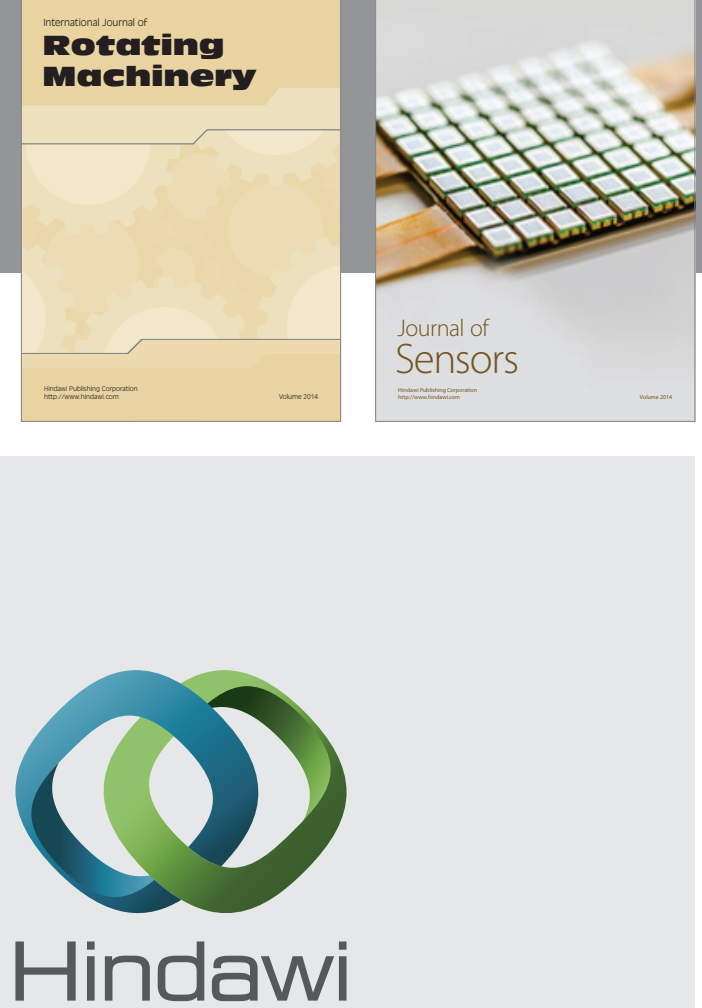

Submit your manuscripts at http://www.hindawi.com
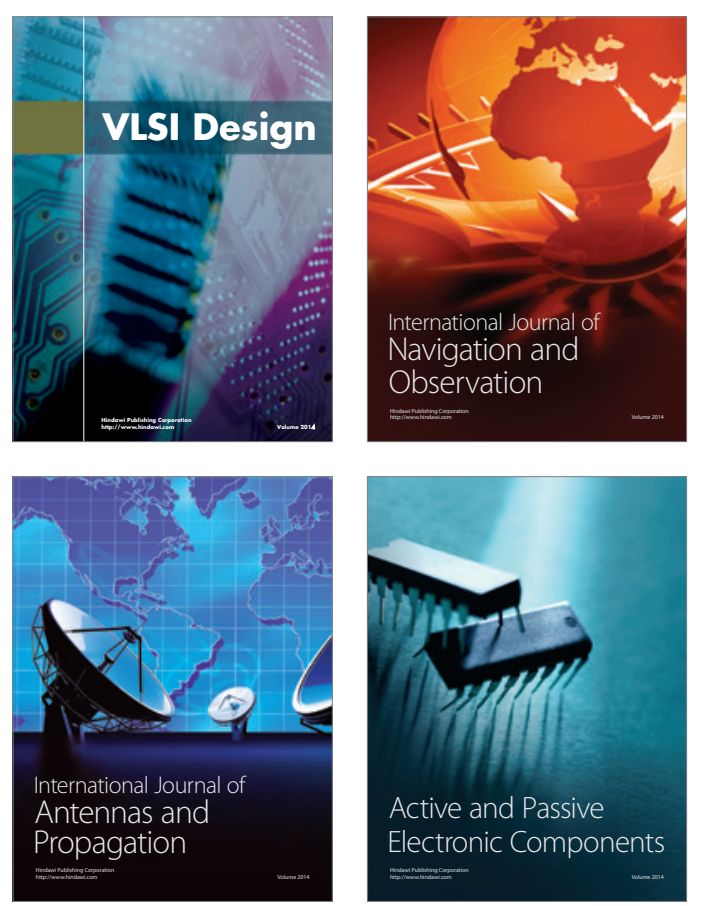
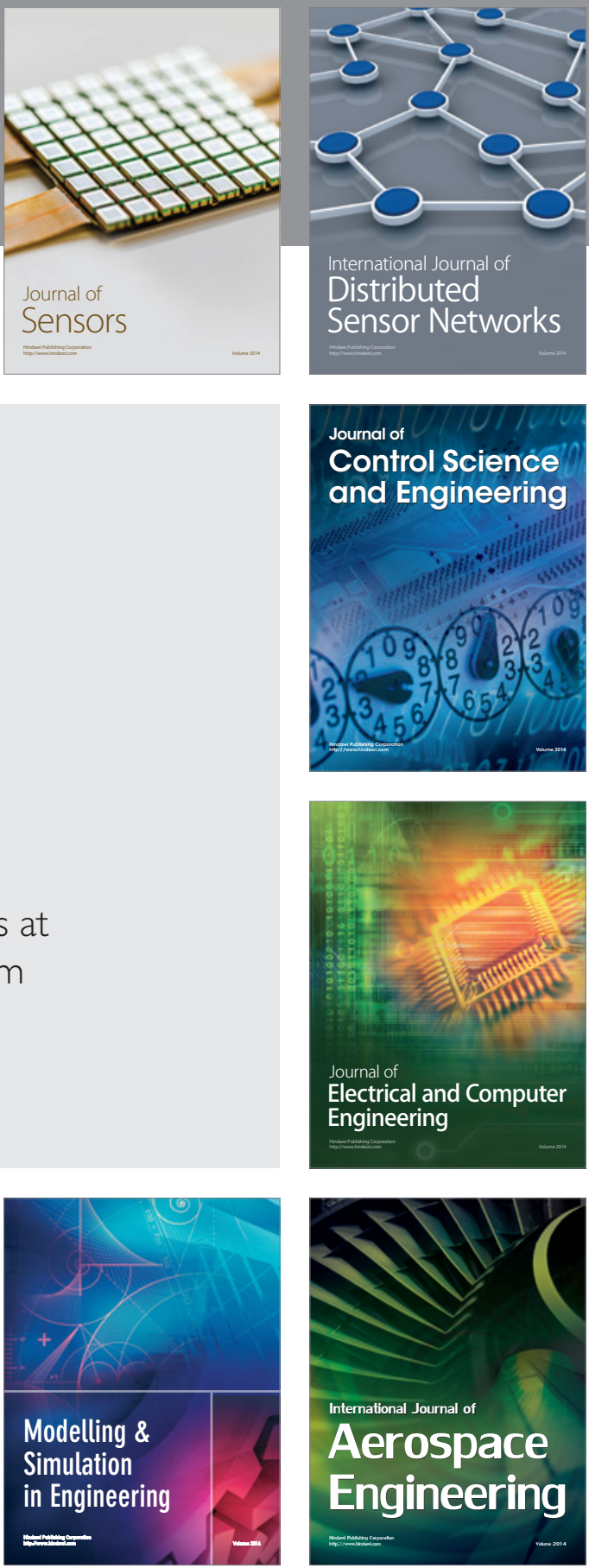

Journal of

Control Science

and Engineering
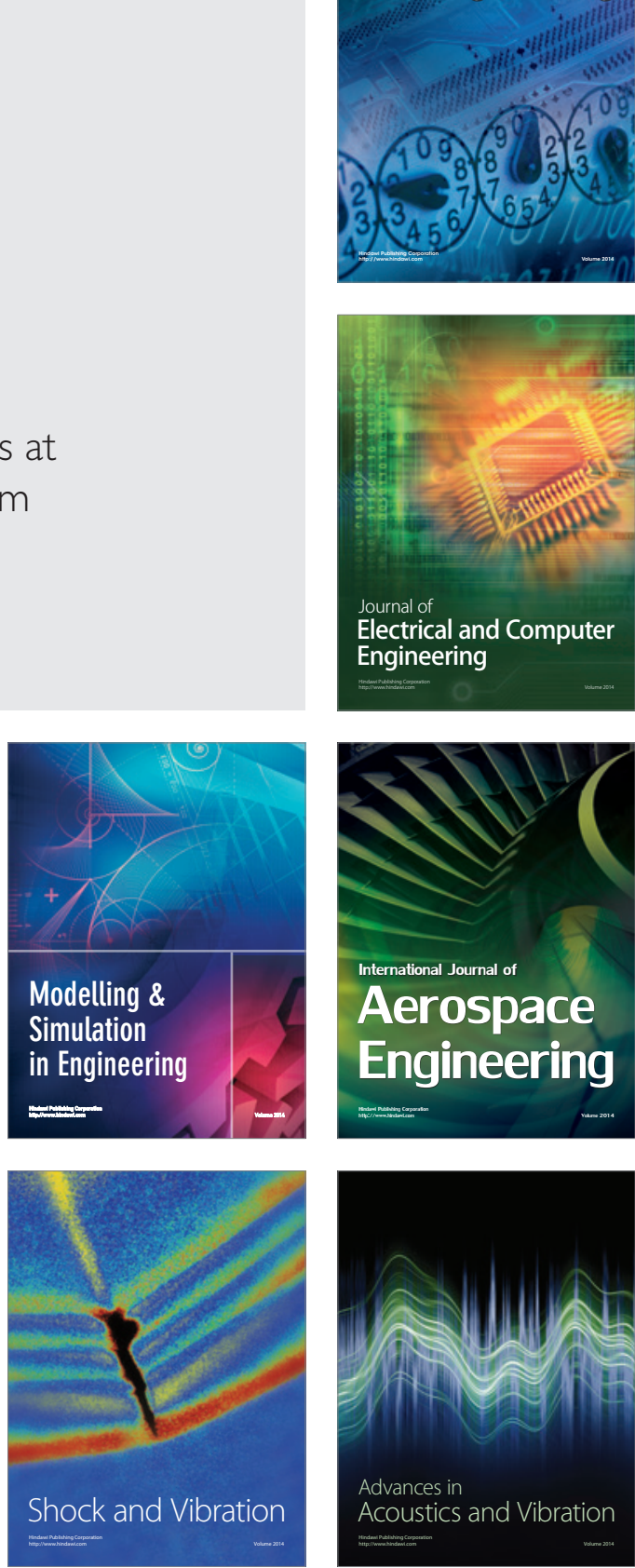